\title{
Computing the Geographic Extent of Maternity Health Services to Predict the Utilization of Skilled Delivery in Siaya County, Western Kenya
}

\author{
Oluoch $F$., \\ Ayodo G.,
}

Department of Public and Community Health, School of Health Sciences, Jaramogi Oginga Odinga University of Science and Technology, Bondo,

Kenya

Owino $F$.,

Department of Spatial Planning, School of Spatial Planning and Natural Resource Management, Jaramogi Oginga Odinga University of Science and Technology, Bondo, Kenya

\section{Okuto E.,}

Department of Applied Statistics, School of Mathematics and Applied Statistics, Jaramogi Oginga Odinga University of Science and Technology, Bondo, Kenya

\section{Doi:10.19044/esj.2020.v16n3p489 ～URL:http://dx.doi.org/10.19044/esj.2020.v16n3p489}

\begin{abstract}
In Kenya, no study has attempted to incorporate the target population, nor the availability coverage and accessibility coverage to expose hidden gaps in the provision of maternity health services that target rural and marginalized populations for a high burden, yet low resource setting such as rural Siaya County. A cross-sectional study design used publicly available geospatial data in combination with administrative ward level data from the web-based district health information software, version 2 (DHIS 2). AccessMod version 5 was used for geographic coverage analysis. ArcGIS (version 10.5) and R (version 3.5.3) sufficed for the preparation of input geospatial data and the manipulation of AccessMod results respectively. The association between the geographic coverage and skilled delivery was computed using a Zero-inflated Poisson regression model at a 95\% confidence level. The findings in Siaya County revealed a higher likelihood of a skilled delivery $34 \%$ (0.34; CI: 0.339-0.347) and $16 \%$ (0.16; CI: $0.162-0.167)$ respectively. This likelihood is for every unit increase in the proportion of pregnant women within a one-hour geographic extent of a hospital and health center - on foot, as compared to being within a similar geographic catchment area of a dispensary $7 \%$ (0.07; CI: $0.0678-$
\end{abstract}


0.0723 ) based on motorcycle traveling time. The immediate implication is that the population coverage capacity and, by extension, quality of existing facilities offering free maternity health services increases a pregnant woman's likelihood of utilizing skilled delivery, regardless of proximity. Future research should also consider looking at the cost-implications of scaling up existing maternity health services, albeit based on local routine health facility data.

Keywords: DHIS2, Geographic Information Systems, Health Services Coverage, Skilled Delivery, Tanahashi's Framework

\section{Introduction}

By 2030, no country should have an MMR greater than 140 maternal deaths per 100,000 live births, a number twice the global target. Yet an estimated $99 \%$ of all maternal deaths occur in developing countries and more than half occur in sub-Saharan Africa, while a nearly one-third occurrence is in South Asia (Kieny et al., 2017; Tangcharoensathien, Mills, \& Palu, 2015; You et al., 2015). According to WHO, the high number of maternal deaths in some areas of the world reflects disparities in access to health services, and highlights the gap between wealth quintiles (WHO \& UNICEF, 2015). In particular, Africa has been showing a substantial reduction in maternal and child mortality. However, a list published by WHO in 2015 showed that the continent is still progressing slower than other regions and contains 25 of the 30 lowest ranked countries to give birth in (WHO \& UNICEF, 2015).

In Kenya, for instance, inequities in maternal mortality continue to persist among population subgroups, and between the rich and the poor $(\mathrm{MoH}$, 2016). For example, only 15 out of 47 counties accounted for $98.7 \%$ of the total MMR of 362 deaths per 100,000 live births in the country (UNFPA, 2014). Yet Siaya, the worst of six counties in the Nyanza region, Western Kenya, with an unacceptably high MMR of 691, also received the best ranking in terms of its overall readiness to provide maternity health services according to a previous service availability and readiness assessment mapping (SARAM) around the same period (Gok, 2014). Thus, close attention must be paid to the measurement tools, techniques, and types of databases used in equity analysis, including how maternity health services coverage has been measured and interpreted (Banke-Thomas, Wright, \& Collins, 2019).

Estimating maternity health services coverage, on the other hand, requires knowledge of the size of the target population (women in need of delivery services) and how many of the target population have been reached with such services. Thus, the domains of Tanahashi's framework for effective coverage (Tanahashi, 1978) will underpin the research and analysis of findings in the current study. Tanahashi's model of health service access encompasses different domains or dimensions including availability, accessibility, 
acceptability, contact or utilization, and effective coverage. Effective coverage, being the objective, is defined as the proportion of the population in need of an intervention that receives an effective intervention. Thus, this is a definition that is posed in equity terms since its denominator is need (O'Connell \& Sharkey, 2013; Tanahashi, 1978). Tanahashi's model is especially useful for an equity analysis because it facilitates the identification of groups with unmet needs (Frenz \& Vega, 2010). This is because maternity health services in Siaya County may not be available, may be difficult to access, or may be unacceptable to certain groups. Such groups are explained to be "missing" or "hidden" from the system. These hidden groups are also explained to represent the inequity of the health system (Frenz \& Vega, 2010).

Moreover, in terms of health system performance, the spatial elements of availability and accessibility can be converted to availability and accessibility coverages. An availability coverage demonstrates what resources are available and in what amount for delivering services (Munoz \& Källestål, 2012). The availability of such resources limits the maximum capacity of the service and thus determines the amount of maternity health services that can be provided to the target population in Siaya County, for instance. Availability coverage relates the capacity of the health system to the size of the target population. An accessibility coverage, on the other hand, determines how physically accessible resources are for the hard-to-reach population (Munoz \& Källestål, 2012; Ray \& Ebener, 2008; Tanahashi, 1978). In other words, independent analysis of availability and accessibility of maternity health services provides a one-dimensional perspective: availability coverage describes how the supply of care is spatially distributed without considering if this supply is physically accessible. Conversely, accessibility coverage looks at how physically accessible a service is to the population without considering if the supply of care is sufficient to cover the demand. Hence, when availability and accessibility coverages are combined in a single analysis, one can define 'geographic (or spatial) coverage' of maternity services for similar high priority settings like Siaya County, Kenya (Munoz \& Källestål, 2012; Ray \& Ebener, 2008).

More notably, with relevant routine data like the total catchment area population for each health facility, and the skilled delivery coverage across every administrative ward level from the web-based DHIS2 platform, the impact of 'geographic coverage' on local utilization of maternity health services can be analyzed in existing GIS tools (Molla et al., 2017). AccessMod platform, for instance, is a free and open source WHO-developed spatial model (WHO, 2016a), which enables measurement of a geographic coverage to help decision makers identify the number and location of pregnant women who will never contact the health system or do so infrequently, even if such groups are not included in routine statistics on utilization (Frenz \& Vega, 2010). 
Therefore, to estimate whether sufficient resources are available to provide maternity health services for at least $90 \%$ of pregnant women in the rural areas of Siaya County, the extent of the catchment area of each facility was calculated by taking into account the location and the maximum coverage capacity of each facility offering free maternity health services, the geographical distribution of the target population, the landscape through which the pregnant women need to cross to reach the health facility, and the mode of transportation. In particular, the current study aimed to find out whether or not there was a significant statistical relationship between skilled delivery coverage and the capacity of maternity health services in the rural parts of Siaya County and its 30 administrative wards.

\section{Materials and Methods \\ Study Area}

Siaya is one of the six counties in the Nyanza region of Kenya. It has a land surface area of approximately $2,530 \mathrm{~km}^{2}$ and water surface area of approximately $1,005 \mathrm{~km} 2$. It approximately lies between latitudes $0^{\circ} 26^{\prime}$ South to $0^{\circ} 18^{\prime}$ North and longitudes $33^{\circ} 58^{\prime}$ and $34^{\circ} 33^{\prime}$ East. The county consists of six sub-counties and thirty wards. Alego Usonga, Bondo, and Gem sub counties have six wards each; Rarieda, Ugenya, and Ugunja subcounties have five, four, and three wards respectively. Alego Usonga is thus the largest subcounty with an approximate area of $605.8 \mathrm{~km} 2$, while Ugunja is the smallest with an approximate area of $200.9 \mathrm{~km} 2$ (Siaya County, 2018).

The female reproductive population was estimated at 222,846 representing $22.7 \%$ of the population and was projected to increase to 238,527 and 273,276 persons in 2022 and 2030 respectively. The county's population density of 318 per square kilometer is further anticipated to increase to 415 and 476 in 2022 and 2030 respectively. In terms of the population distribution by residence, $89 \%$ of Siaya County residents currently live in the rural areas. As of 2017 , the county had $434.2 \mathrm{~km}$ of bitumen standard roads, $1297.41 \mathrm{~km}$ of gravel, $532.78 \mathrm{~km}$ of earth and a further 1,170 narrow roads. The main development challenges include poor infrastructure, inadequate water supply, erratic electricity supply, high prevalence of HIV/AIDS, and high unemployment. As a result, about $47.5 \%$ of the total population in the county currently lives below the poverty line (Siaya County, 2018).

Moreover, by 2018, the number of health facilities registered in the county was 220, comprising 11 level 4 hospitals, 50 level 3 health centers, and 159 level 3 dispensaries. This was an increase of $47.6 \%$ from 149 health facilities (seven hospitals, 32 health centers, and 110 dispensaries) in 2013 (Nyangueso, Hayombe, \& Owino, 2018). The county's uptake of antenatal care and skilled birth attendance was higher than the national average, but it is not certain whether all the demand was met within or there was a spill over to 
neighboring counties (Nyangueso et al., 2018). According to the last demographic and Health Surveys (KDHS), the percentage of mothers receiving antenatal care from a skilled provider in the county was $98.5 \%$ as compared to $95.5 \%$ nationally (KDHS, 2014). Similarly, the percentage of mothers who delivered in a health facility was $69.6 \%$ compared to Kenya's $61.2 \%$. Demand for the maternal services by 2017 was 38,449 annually, synonymous with the distribution of livebirths in Figure 1 below (Nyangueso et al., 2018).

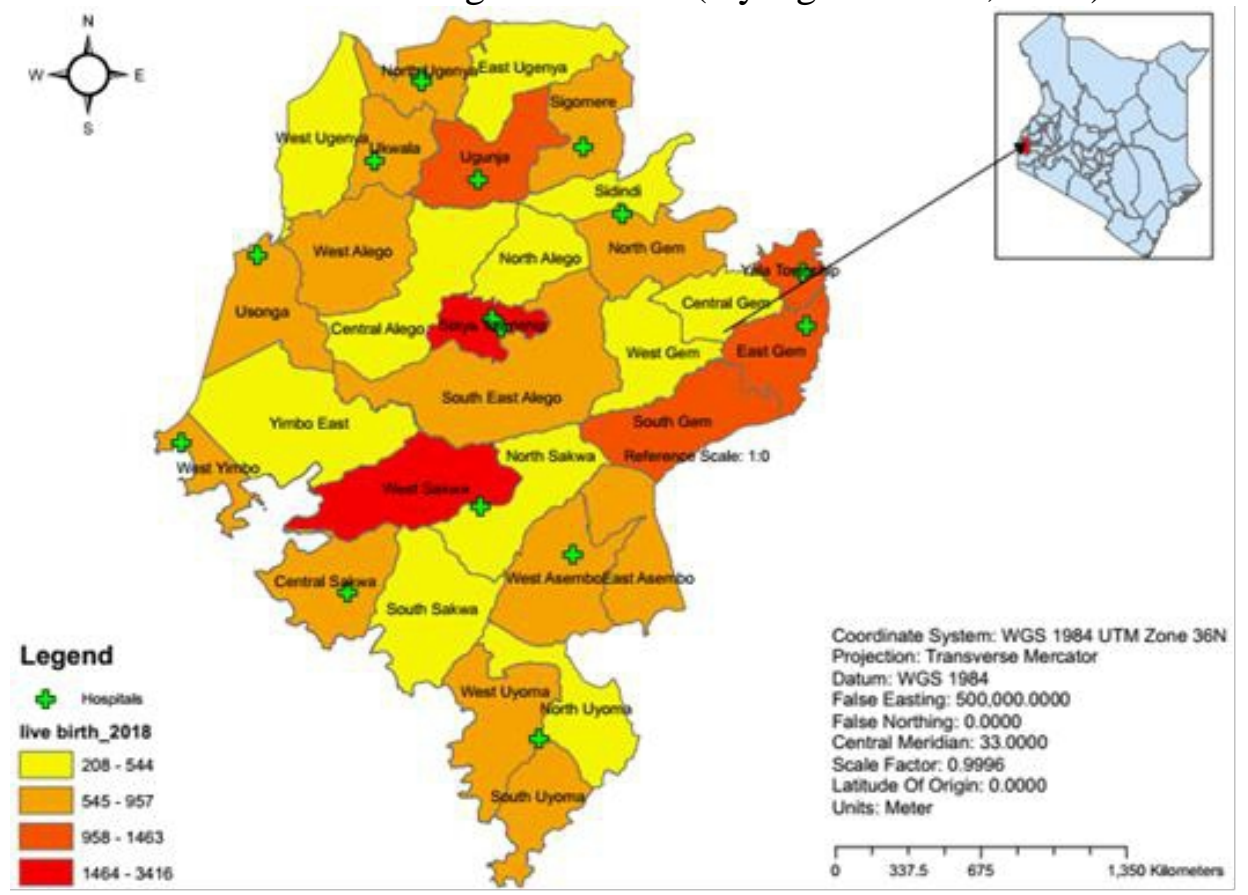

Figure 1. A map showing the livebirths per administrative ward and the location of hospital facility types offering free maternity services across Siaya County.

\section{Study Design}

The current cross-sectional study used publicly available geospatial data in combination with routine health facility data at the ward administrative level from the web based DHIS2 platform. The catchment area for different levels of maternity health services was simulated using a least-cost algorithm based on Tanahashi's framework (1978) of evaluating health service coverage (Ebener \& Stenberg, 2016; Munoz \& Källestål, 2012; Ray \& Ebener, 2008; Schmitz et al., 2019; WHO, 2016).

A Zero-Inflated Poisson regression model was then used to check if indeed there was a significant statistical relationship between skilled delivery coverage and geographic coverage of maternity health services in the rural parts of Siaya County based on walking and/or motorbike traveling time, albeit disaggregated by level of care. The target population consisted of all pregnant women living within the administrative boundaries of mainland Siaya County 
in 2018. The study inclusion criteria consisted of only pregnant women who delivered in Tier two and three facilities offering free maternity health services. Private and/or for-profit facilities offering maternity health services, including women living outside the administrative boundaries of mainland Siaya County, were excluded from the study.

\section{Geographic Coverage Analysis Software}

Modeling of geospatial data was made possible via AccessMod version 5-an open-source and standalone spatial model developed by the WHO, which is freely accessible through the AccessMod official web site (https://accessmod.org). Also, it comes with a user manual and a sample dataset to guide users on the use of its different modules. Moreover, a thirdparty GIS software, ArcGIS (version 10.5), in combination with a free software environment for statistical computing and graphics, R (version 3.5.3), sufficed for the preparation of input data and the manipulation of AccessMod results respectively.

\section{Sources and Preparation of Input Data}

Geographic coverage analysis using AccessMod 5 required several data sets (see Table 1 below). Both raster and vector data were used as inputs, but the latter were transformed into raster data during the analysis. To mitigate potential resolution issues and ensure compatibility between the different sources of GIS data, and to produce accurate results, it was important that an equal-area projection was used for the data to avoid strong biases in the surface of the modeled catchment areas. It was also necessary that meters were used as map units. This is so that travelled distances are correctly linked to the userdefined travelling speeds expressed in $\mathrm{km} / \mathrm{h}$.

Moreover, all the geospatial datasets in raster format were homogenized in terms of projection and $30 \mathrm{~m}$ spatial resolution of the digital elevation model (DEM) raster. The Universal Transverse Mercator (UTM) projected coordinate system was sufficient (the UTM zone for Siaya County is Zone 36), as the data needed to be projected in a metric system when performing the different geospatial analyses. Populations at the urban areas were spatially excluded using urban extent vector data from Global RuralUrban Mapping Project. This was done towards a more realistic representation of the magnitude of the relationship between existing geographic inequalities and utilization of free maternity health services. All input geospatial datasets were ultimately cropped to the administrative boundaries of mainland Siaya County.

In particular, geographic coverage analysis is required to have the following GIS layers at disposal: 1. Administrative boundaries down to the 
ward level sourced from the Database of Global Administrative Areas (GADM); 2. Geographic point location of all existing facilities offering free maternity health services based on DHIS2, corroborated by the Kenya Master Health Facility List (KMHFL); 3. Road network; 4. Hydrographic network (major rivers and water bodies) were sourced from the United Nations Humanitarian Data Exchange portal; 5. Land use/land cover were sourced from Regional Centre for Mapping of Resources for Development (RCMRD); 6. Digital Elevation Model (DEM) was sourced from Shuttle Radar Topography Mission (STRM) digital elevation model data, at a spatial resolution of 30 meters (1 arc-second), from the U.S. Geological Survey; 7. A 2018 dataset of the spatial distribution of the population for Kenya was obtained from the WorldPop Project (Worldpop, 2019).

Moreover, user defined tabular data for each transportation mode with a corresponding travel speed for every combined land cover class, under each travel scenario (walking and/or being carried on a motor cycle), was specified in line with a recent study in Kigoma Region in Tanzania (Chen et al., 2017). This was important to simulate real-life travel experiences in which travel time may vary by terrains, road types, and means of transportation used. As such, various sources were used to ascertain the transportation-specific travel speed for each land cover type in the dry season, including a motorcycle analysis conducted in Hanoi, Vietnam (Chen et al., 2017; Oanh, Phuong, \& Permadi, 2012).

For routine health facility data, the web based DHIS2 platform sufficed to collect the total catchment area population for each health facility, including the delivery by skilled birth attendant coverage across all 30 administrative wards in Siaya County.

Table 1. Characteristics of the various Input Geospatial Datasets

\begin{tabular}{l|lllll}
$\begin{array}{l}\text { Data } \\
\text { Format }\end{array}$ & Data Layer & Year & Source & $\begin{array}{l}\text { Spatial } \\
\text { resolution }\end{array}$ & $\begin{array}{l}\text { Description/additional } \\
\text { information }\end{array}$ \\
\hline Raster & $\begin{array}{l}\text { Population } \\
\text { Distribution } \\
\text { grid }\end{array}$ & 2018 & WorldPop & Raster & $\begin{array}{l}\text { Spatially explicit distribution of } \\
\text { population over the study area. } \\
\text { The value of each 100-meter } \\
\text { gridded cell represented the } \\
\text { estimated number of people in } \\
\text { an area of 100 square meters in }\end{array}$ \\
Raster & Land use grid & 2016 & $\begin{array}{l}\text { Regional } \\
\text { Centre for } \\
\text { Mapping of } \\
\text { Resources for }\end{array}$ & Raster & $\begin{array}{l}\text { Spatial distribution of the } \\
\text { different categories of land use } \\
\text { on which travelling speed may be } \\
\text { different. }\end{array}$ \\
Raster & $\begin{array}{l}\text { Development } \\
\text { (RCMRD) }\end{array}$ & $\begin{array}{l}\text { Shuttle Radar } \\
\text { Topography } \\
\text { Mission }\end{array}$ & Raster & $\begin{array}{l}\text { Altitude distribution used to } \\
\text { derive slopes and correct } \\
\text { travelling speeds in the case of }\end{array}$ \\
& $\begin{array}{l}\text { Digital } \\
\text { Elevation } \\
\text { Model (DEM) }\end{array}$ & 2010 & &
\end{tabular}




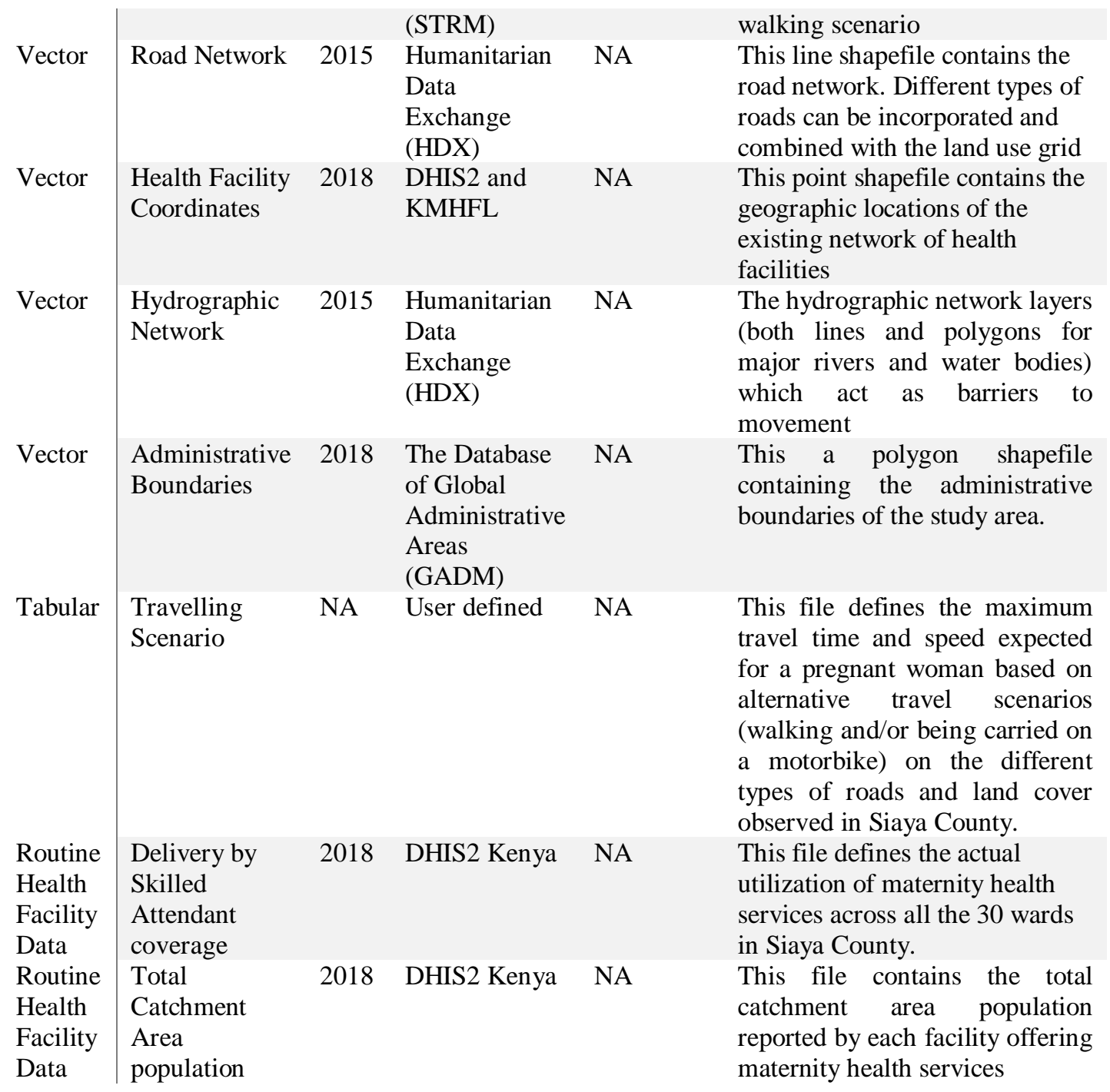

\section{Modeling Geographic Catchment Areas of Maternity Health Services}

To enable the geographic coverage analysis, both river and road network vector datasets were transformed to raster datasets consisting of 30meter gridded cells. Also, they were then overlaid on the land cover raster dataset using the merge cover tool in AccessMod 5.0 toolbox. This created a combined land cover raster dataset with 10 unique land feature classes including 5 road land cover classes. The 5 non-road land cover classes included dense vegetation, medium dense vegetation, low dense vegetation, bare areas, and built areas. For the purposes of analysis, water bodies and rivers were considered to be impassable to any form of transportation. In particular, the geographic coverage analysis integrated the spatial distribution of maternity health services (supply) and of the target population (demand). 
The catchment area of each facility offering maternity health services was calculated by taking into account its total catchment area population, the population distribution layer, the terrain topography, and the travelling scenarios through the different land categories (see Table 2 below). 
Table 2. Travel Speeds per Land Cover Type to the Nearest Maternity Health Services in Siaya County by Travel Scenario

Travel Speeds $(\mathrm{km} / \mathrm{hr})$

\begin{tabular}{l|lllll}
\hline & Land Cover Type & \multicolumn{2}{c}{$\begin{array}{c}\text { Walking } \\
\text { Scenario } \\
\text { (Scenario1) }\end{array}$} & & $\begin{array}{c}\text { Walking + Motorcycle } \\
\text { Scenario } \\
\text { (Scenario 2) }\end{array}$ \\
1 & Dense vegetation & 1 & WALKING & 1 & WALKING \\
2 & Medium dense vegetation & 1.5 & WALKING & 1.5 & WALKING \\
3 & Low dense vegetation & 2 & WALKING & 2 & WALKING \\
4 & Bare areas & 2.5 & WALKING & 2.5 & WALKING \\
5 & Built areas & 2.5 & WALKING & 20 & MOTORIZED \\
6 & Bound surface & 2.5 & WALKING & 40 & MOTORIZED \\
7 & Loose surface & 2.5 & WALKING & 25 & MOTORIZED \\
8 & Dry weather road & 2.5 & WALKING & 35 & MOTORIZED \\
9 & Main track motorable & 2.5 & WALKING & 50 & MOTORIZED \\
10 & Other tracks and footpaths & 2.5 & WALKING & 2.5 & WALKING
\end{tabular}

The extent of the catchment area was thus determined by either the travel time or the catchment population, whichever was reached first. This means that health facilities offering maternity services that had realized the maximum travel time, but not their maximum capacity, were working below their capacity. On the other hand, health facilities that had reached their catchment population before reaching one hour of travelling time were operating at their maximum capacity. The model utilized the least-cost algorithm whereby the location of a facility offering maternity health services was selected as the origin. Consequently, the maximum travel time of 60 minutes (in line with Ministry of Health) was also considered as the limitation for determining the extension of the corresponding catchment area. The model assumes that a patient can only be served by one facility offering maternity services and that mainland Siaya County is a closed system (i.e., the population cannot be served by health facilities outside Siaya County and a population outside the County cannot seek care in it).

Moreover, a vector layer that describes the extent of each facility offering maternity health services was outputted for each of the 2 travel scenarios (walking and/or motorbike). Thus, each scenario-specific geographic coverage vector layer was represented by a single shapefile based on either of both transportation scenarios (walking and/or motorbike) and health facility types (dispensary, health centers or hospital). As such, all 1-hour service catchment areas for each corresponding travel scenario were mapped in ArcGIS 10.5 to show the extent of the catchment areas within which one can 
get access to maternity services in less than 1 hour.

Furthermore, the zonal statistics option in AccessMod 5 was used to calculate geographic coverage $(\%)$ for each of the 30 wards in Siaya County using the corresponding administrative boundaries vector layer. Therefore, geographic coverage was calculated using the total number of pregnant women per administrative ward level as the denominator, and the proportion of pregnant women within 1 hour of travel time of a facility offering maternity health services with enough capacity to cover the demand in each ward administrative level as the numerator. More specifically, the estimated total number of people per grid-cell was used as a proxy measurement for the distribution of pregnant women in Siaya County, which is the target population of maternity health services, since expected births or pregnancies comprise 0.04 (4\%) of the total population annually (Republic of Kenya, 2014b).

\section{Estimating the Likelihood of Skilled Birth Attendance based on Facility Capacity}

Delivery by Skilled Birth Attendant Coverage was the main outcome variable. Thus, this indicator was calculated using the number of women who actually delivered in a health facility as the numerator and the total estimated deliveries as the denominator, albeit for each health facility type (dispensary, health Center or hospital) and across all the 30 administrative wards in Siaya County.

Zero inflated Poisson regression was used to model the statistical relationship between geographic coverage by walking/motorbike traveling time scenario (the independent variable) and Delivery by Skilled Birth Attendant Coverage (dependent variable) at the ward administrative level, albeit for each health facility type (dispensary, health centers or hospital). The zero-inflated model was considered due to the excess number of zero cases of skilled delivery coverage, since pregnant women lack access to and/or prefers a particular facility types in some administrative wards in Siaya County.

The model was formulated as follows:

Suppose that for each administrative ward level number of skilled deliveries ( $\left.Y_{i}\right)$, there are two possible outcomes for cases. The first case occurs when the count is zero. Nevertheless, the second case is generated when non-zero counts are registered and occurs with the probability $(1-\pi)$ assuming that the first case occurs with a probability of $\pi$ where $0<\pi<1$. Thus, the probability of zero-inflated Poisson variable $y_{i}$ can be written as: 


$$
\operatorname{pr}\left(Y_{i}=j\right)=\left\{\begin{array}{l}
\pi_{i}+\left(1-\pi_{i}\right) \exp \left(-\mu_{i}\right), \text { if } j=0 \\
\left(1-\pi_{i}\right) \frac{\mu_{i}^{y_{1}} \exp \left(-\mu_{i}\right)}{y !}, \text { if } j>0
\end{array}\right.
$$

Where $\mu_{\mathrm{i}}$ is a rate parameter expressing the rate of ward level skilled deliveries.

The likelihood function prob. $\left(\mathrm{Y}_{\mathrm{i}}=\mathrm{j}\right)$ was then used as the family of the generalized linear model $(\mathrm{glm})$. Moreover, for the zero inflated model, maximum likelihood estimation method was used to obtain the slope parameter estimates. Significance of the slope parameters were assessed using probability (p) value computed at $95 \%$ confidence level.

\section{Ethical Considerations}

In close collaboration with the Siaya County Health Department, Division of Reproductive Health, the current study protocol was approved by the the Jaramogi Oginga Odinga University Ethical Review Board.

\section{Results}

\section{Summary Statistics from DHIS2: Skilled Delivery Capacity Utilization}

According to data from the web based DHIS2 platform, the total capacity of all health facilities for skilled deliveries across all administrative wards in Siaya County in 2018 was 39,419, yet only 25,755 (66\%) of this capacity was effectively utilized. This was despite almost half (14/30) of the administrative wards in the county utilizing less than $50 \%$ of their estimated capacity for skilled deliveries during the same period. In particular, the worst performing administrative ward units were South Sakwa (28\%), Central Alego (24\%), Central Gem (23\%), North Uyoma (21\%), even if Siaya Township (237\%), Yala Township (138\%), Usonga (129\%), and West Sakwa (115\%) Wards recorded more than $100 \%$ utilization of their skilled delivery capacity as depicted in Figure 2 below. 


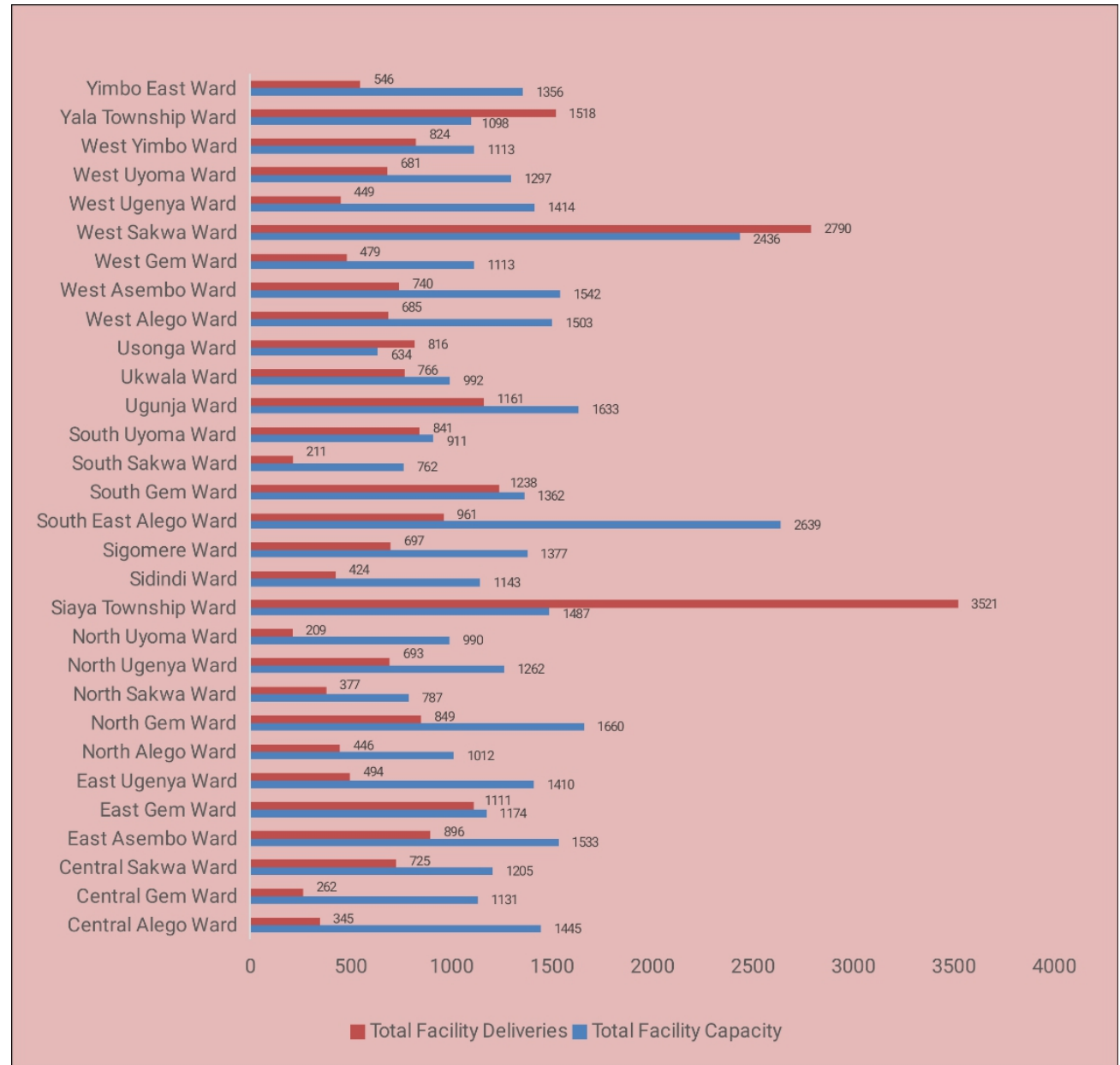

Figure 2. The total number of facility deliveries versus the skilled deliveries capacity across all wards in Siaya County.

Moreover, in terms of total capacity by health facility type, Dispensaries represented the largest proportion at $41 \%$ and Health Centres followed closely at $34 \%$. This is despite Hospitals having 22\% and Medical Clinics representing the least proportion at only $3 \%$ of the estimated skilled delivery capacity in Siaya County. Nevertheless, Hospitals utilized nearly one and a half times (149\%) their estimated skilled delivery capacity and Health Centres on the other hand utilized $68 \%$ of their estimated capacity. This is despite Dispensaries and Medical Clinic facility types utilizing only $20 \%$ and 19\% of their estimated capacity respectively, as illustrated in Figures 3 and 4 below. 


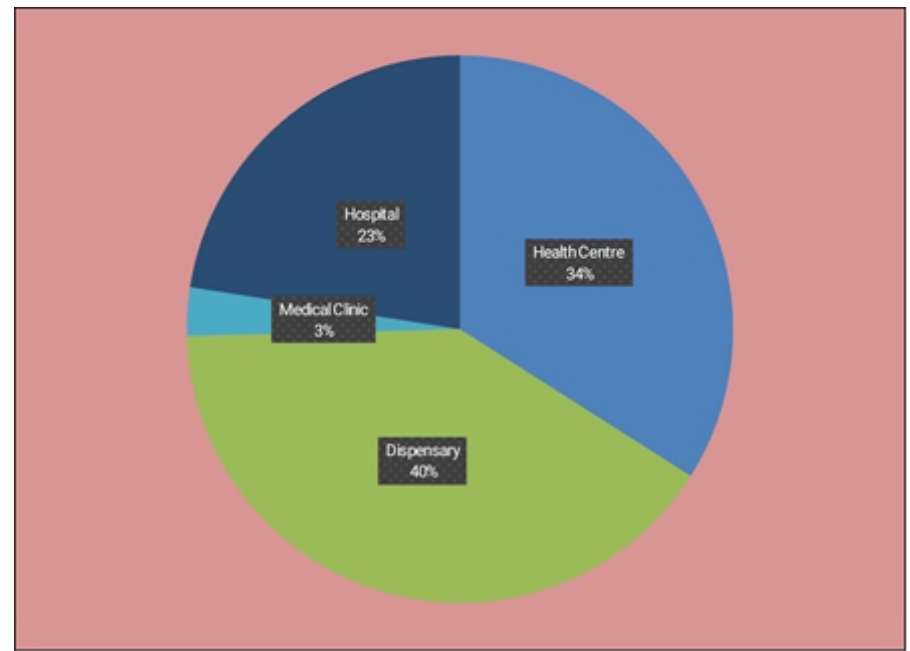

Figure 3. Showing the estimated skilled deliveries capacity by health facility type across all wards in Siaya County

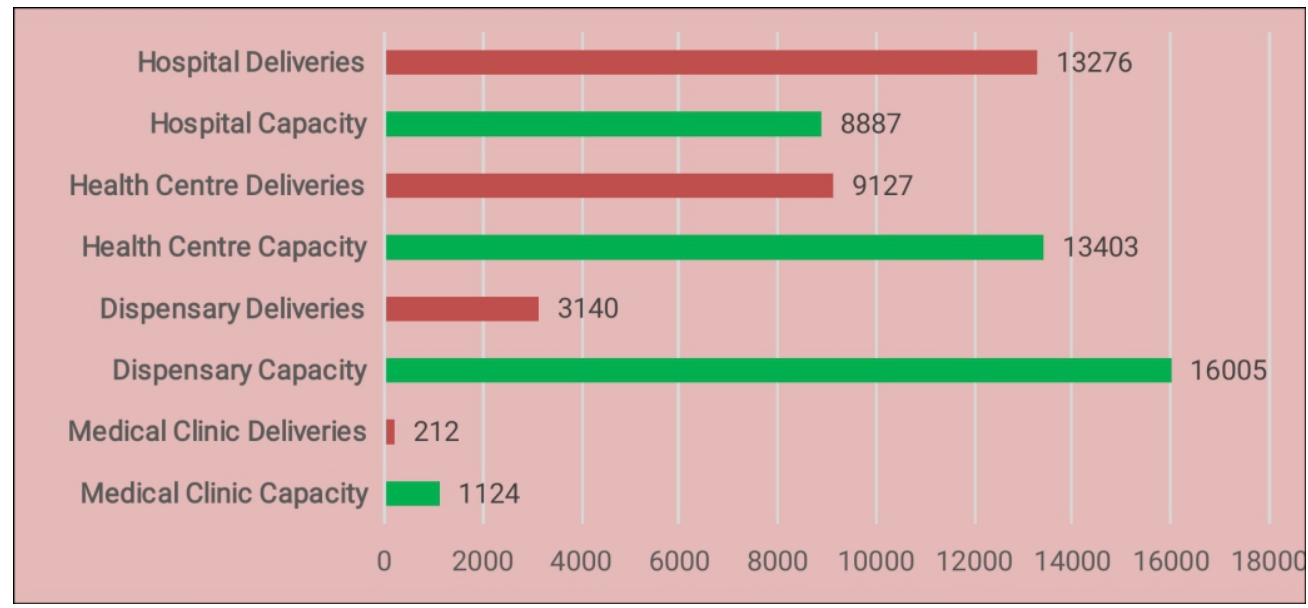

Figure 4. Showing the total deliveries versus the skilled deliveries capacity across all facility types in Siaya County

Furthermore, in terms of capacity by facility ownership, health facilities owned by the Ministry of Health had the largest share of the total skilled delivery capacity at $89 \%$. This is despite Faith Based Organizations and Private facilities having only $7 \%$ and $4 \%$ of the estimated skilled delivery capacity in Siaya County respectively, as illustrated in Figure 5 below. 


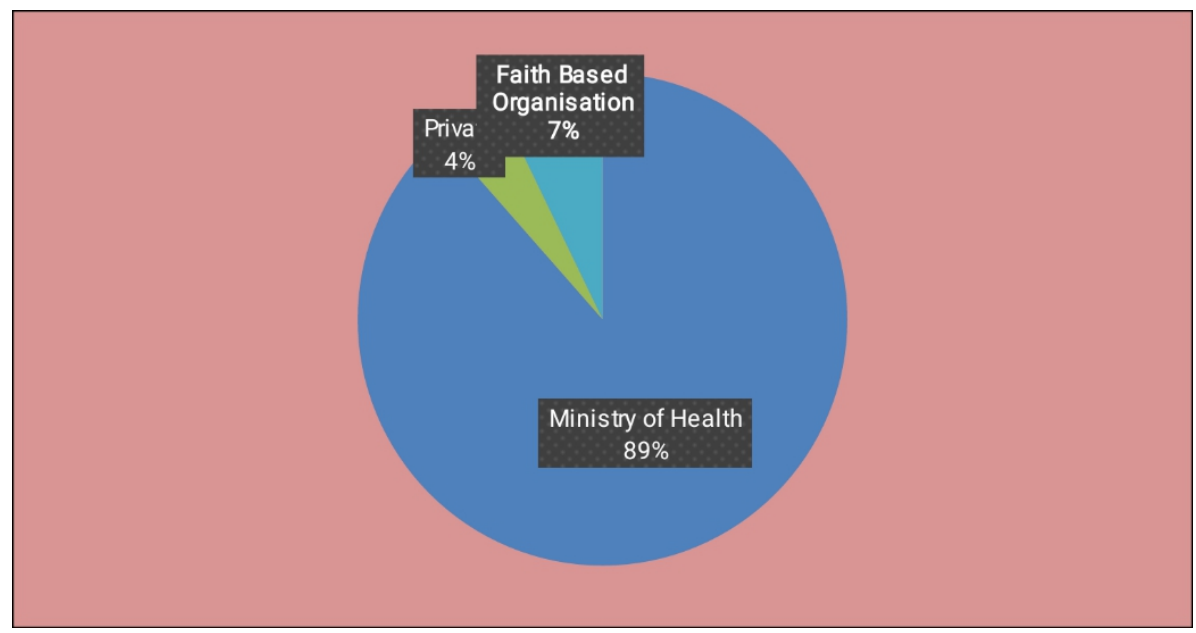

Figure 5. Comparing the capacities of health facilities by ownership in Siaya County

\section{Geographic Coverage Analysis of Facilities Offering Maternity Health Services}

The current geographic coverage analysis involved 157 facilities; 97 dispensaries, 46 health centers, and 14 hospitals offering free maternity services in Siaya County as shown in the Table 3 below. The geographic coverage analysis was able to model the extension of the catchment area of each health facility type. In this analysis, the population coverage capacity of each health facility was considered as the size of the supply, and the population distribution grid as the spatial distribution of the demand.

Table 3. Geographic Coverage (\%) by Facility Type and Travel Scenario across Siaya County

\begin{tabular}{l|ll}
$\begin{array}{l}\text { Facility Type } \\
\text { No. }(\%)\end{array}$ & $\begin{array}{l}\text { Walking Scenario } \\
\text { (Scenario 1) } \\
\text { No. }(\%)\end{array}$ & $\begin{array}{l}\text { Walking + Motorcycle } \\
\text { Scenario } \\
\text { (Scenario 2) } \\
\text { No. (\%) }\end{array}$ \\
\hline $\begin{array}{l}\text { Dispensaries } \\
\begin{array}{l}\text { 97 (62) } \\
\text { Health Centers }\end{array}\end{array}$ & $8,833(23)$ & $11,737(31)$ \\
$\begin{array}{l}\text { 46 (29) } \\
\text { Hospitals }\end{array}$ & $5,146(14)$ & $9,117(24)$ \\
$14(9)$ & $1,797(5)$ & $5,666(15)$
\end{tabular}

For geographic coverage analysis of dispensary facility types based on the walking scenario, less than $20 \%$ of pregnant women in 13/30 wards were covered by a dispensary with enough capacity to address the demand. This is despite the fact that more than 35\% of pregnant women in Central Alego, North Ugenya, and South East Alego wards were within an hour of walking to a dispensary with enough capacity to cover the demand. Moreover, when scenario 2 (walking + motorbike) was considered, the situation was slightly 
improved with more than 35\% of pregnant women in 10/30 wards in Siaya County having the ability to reach a dispensary with enough capacity to cover the demand within an hour of traveling with a motorcycle, despite insufficient resources to provide maternity health services to more than $80 \%$ of pregnant women in 7/30 wards even if they opted for motorcycle transport. In summary, considering the best case (motorized) scenario, at least 56 dispensaries were found to be working above their capacity, yet only $23 \%$ of pregnant women across Siaya County could access a dispensary with enough capacity to cover the demand within an hour of walking. Also, only $31 \%$ could do the same if they had access to motorcycle transport as shown in Figure 5 below.

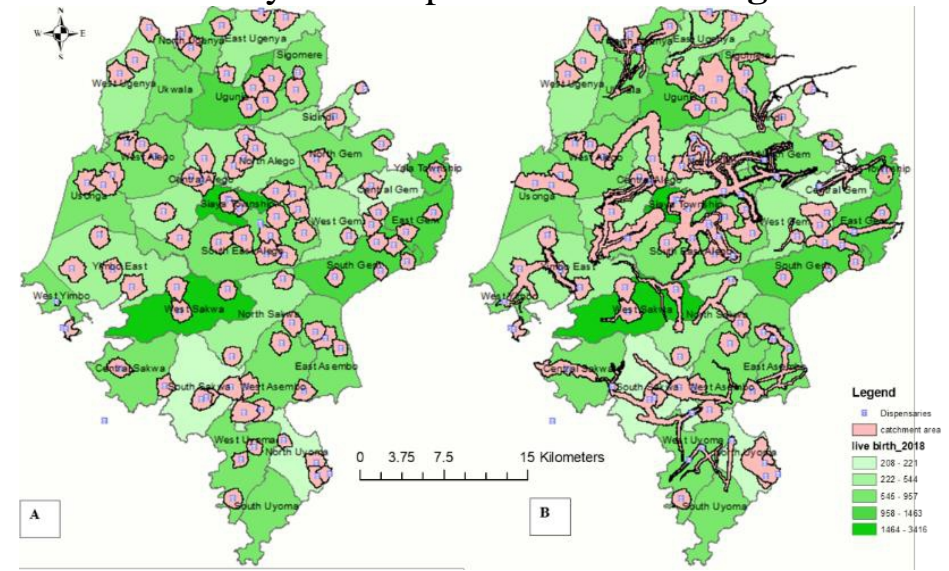

Figure 5. The distribution of dispensary level geographic coverage across all wards in Siaya county $(\mathrm{A}=$ walking scenario; $\mathrm{B}=$ motorized scenario $)$.

For geographic coverage analysis of health center facility types based on walking scenario, less than 5\% of pregnant women in 9/30 wards were covered by a health center with enough capacity to cover the demand. This is despite the fact that more than 30\% of women in Yala Township, Central Gem, East Asembo, Sidindi and South Gem ward were within an hour of walking to a health center with enough capacity to cover the demand. Moreover, when scenario 2 (motorcycle) was considered, more than $40 \%$ of pregnant women in $7 / 30$ wards were covered by a health center with enough capacity to address the demand within an hour of traveling time with a motorcycle, despite insufficient resources to provide maternity health services to more than $90 \%$ of pregnant women in $8 / 30$ wards even if they opted for motorcycle transport as illustrated in Figure 6 below. 


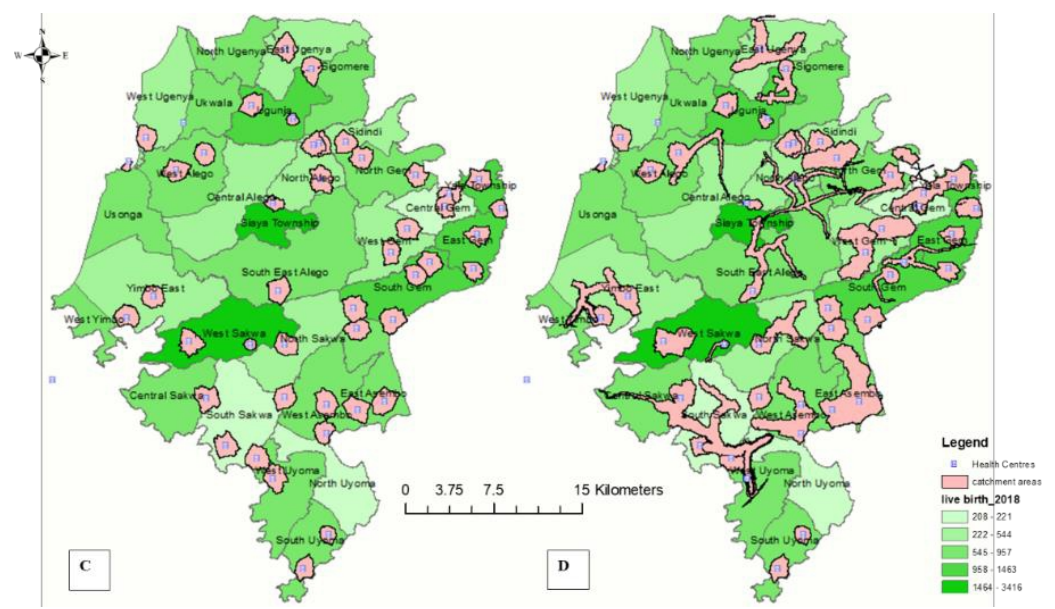

Figure 6. The distribution of health center level geographic coverage across all wards in Siaya County $(\mathrm{C}=$ walking scenario; $\mathrm{D}=$ motorized scenario $)$.

In summary, considering the best case (motorized) scenario, at least 21 health centers were found to be working over their capacity, yet only $14 \%$ of pregnant women across Siaya County could access a health center with enough capacity to cover the demand within an hour of walking, while only $24 \%$ could do the same if they opted for motorcycle transport.

Table 4. Accessibility Coverage (\%) by Facility Type and Travel Scenario across all administrative wards Siaya County

Geographic Coverage (\%)

\begin{tabular}{l|lllllll}
\hline $\begin{array}{l}\text { Administrative } \\
\text { Wards }\end{array}$ & $\begin{array}{c}\text { Estimated } \\
\text { Deliveries }\end{array}$ & \multicolumn{2}{c}{ Dispensaries } & & Health Centers & & Hospitals \\
Central Alego & 1446 & 36.7243 & 53.1420 & 0.2991 & 2.8826 & 0.0000 & 0.9180 \\
Central Gem & 2262 & 24.7715 & 32.0114 & 31.1263 & 51.7271 & 0.0000 & 17.2069 \\
Central Sakwa & 1125 & 10.9274 & 26.2879 & 11.3804 & 25.9232 & 11.7858 & 45.3591 \\
East Asembo & 1533 & 20.1721 & 25.2263 & 32.2737 & 68.0517 & 0.0000 & 0.0000 \\
East Gem & 2349 & 14.8871 & 19.9390 & 25.1572 & 30.2818 & 16.5921 & 17.6026 \\
East Ugenya & 1408 & 19.8558 & 30.0388 & 11.9274 & 26.6227 & 0.0000 & 0.3225 \\
North Alego & 1011 & 19.5951 & 49.5604 & 23.1965 & 29.0678 & 5.9802 & 19.7582 \\
North Gem & 3319 & 21.3900 & 25.9949 & 19.3017 & 42.4755 & 2.5330 & 10.6832 \\
North Sakwa & 722 & 15.2934 & 18.7588 & 11.7306 & 28.0217 & 0.0000 & 1.7428 \\
North Ugenya & 1264 & 37.6092 & 43.8519 & 0.0182 & 1.1459 & 20.6128 & 43.6795 \\
North Uyoma & 991 & 30.2256 & 42.2229 & 1.6436 & 4.5327 & 2.2929 & 8.2192 \\
Siaya & 1503 & 28.8157 & 62.2486 & 0.0020 & 9.6421 & 14.4055 & 38.1067 \\
Township & 2286 & 17.4045 & 24.4829 & 33.3506 & 43.6518 & 4.5589 & 9.0545 \\
Sidindi & 2710 & 25.2825 & 37.0157 & 2.3448 & 2.7953 & 15.4941 & 39.0575 \\
Sigomere & 2638 & 49.3891 & 57.8603 & 4.3031 & 13.7512 & 0.0000 & 20.2491 \\
South East & & & & & & & Malking \\
Alego & & & & & & & \\
\end{tabular}




\begin{tabular}{l|lllllll} 
South Gem & 2805 & 20.8962 & 19.8834 & 35.4040 & 36.0925 & 0.0000 & 0.0000 \\
South Sakwa & 674 & 17.2918 & 28.2095 & 23.9874 & 41.5043 & 0.0000 & 17.1651 \\
South Uyoma & 911 & 0.0000 & 1.5124 & 23.1900 & 23.7121 & 2.8163 & 4.2013 \\
Ugunja & 3509 & 33.1629 & 46.8538 & 13.6429 & 26.8777 & 7.8966 & 14.4243 \\
Ukwala & 992 & 0.0000 & 9.5924 & 0.8999 & 1.1483 & 18.3962 & 32.0444 \\
Usonga & 633 & 26.2401 & 26.0321 & 3.4834 & 3.6935 & 19.3980 & 25.3118 \\
West Alego & 1502 & 22.8716 & 34.1598 & 15.2982 & 21.8603 & 0.0000 & 0.0000 \\
West Asembo & 1541 & 31.4889 & 33.4515 & 19.6674 & 30.2992 & 8.0024 & 8.6160 \\
West Gem & 1730 & 10.1842 & 19.5064 & 15.6608 & 43.2690 & 0.0000 & 0.0793 \\
West Sakwa & 2313 & 13.6176 & 20.3745 & 16.1652 & 23.1096 & 5.4348 & 28.4101 \\
West Ugenya & 1413 & 24.6747 & 24.8392 & 7.7048 & 7.9437 & 0.0789 & 13.2875 \\
West Uyoma & 1296 & 15.9486 & 20.8122 & 22.3501 & 25.2758 & 5.3383 & 29.2326 \\
West Yimbo & 1057 & 20.5761 & 41.9235 & 0.0000 & 13.6333 & 12.9145 & 13.6700 \\
Yala Township & 2196 & 4.6479 & 18.7836 & 30.5314 & 60.3890 & 12.7717 & 53.1741 \\
Yimbo East & 1287 & 30.8417 & 35.4253 & 12.2589 & 24.6156 & 0.0000 & 10.7419
\end{tabular}

For geographic coverage analysis of hospital facility types based on walking scenario, less than $5 \%$ of pregnant women in half (15/30) of the wards in Siaya County were covered by a hospital with enough capacity to address the demand, even if 11/30 wards achieved a geographic coverage of between 11 and $20 \%$ under the same scenario. The situation was slightly better when scenario 2 (motorcycle) was considered, since between 20 to 53\% of pregnant women in 10/30 wards could access a hospital with enough capacity to cover the demand within an hour of traveling time with a motorcycle. This is despite insufficient resources to provide maternity health services to more than $90 \%$ of pregnant women in 11/30 wards even if they opted for motorcycle transport as shown in Figure 7 below. In summary, considering the best case (motorized) scenario, at least 8 hospitals were found to be working beyond their estimated capacity, yet only 5\% of pregnant women in Siaya County could access a hospital with enough capacity to cover the demand within an hour of walking. On the other hand, only $15 \%$ could do the same if they opted for motorcycle transport. 


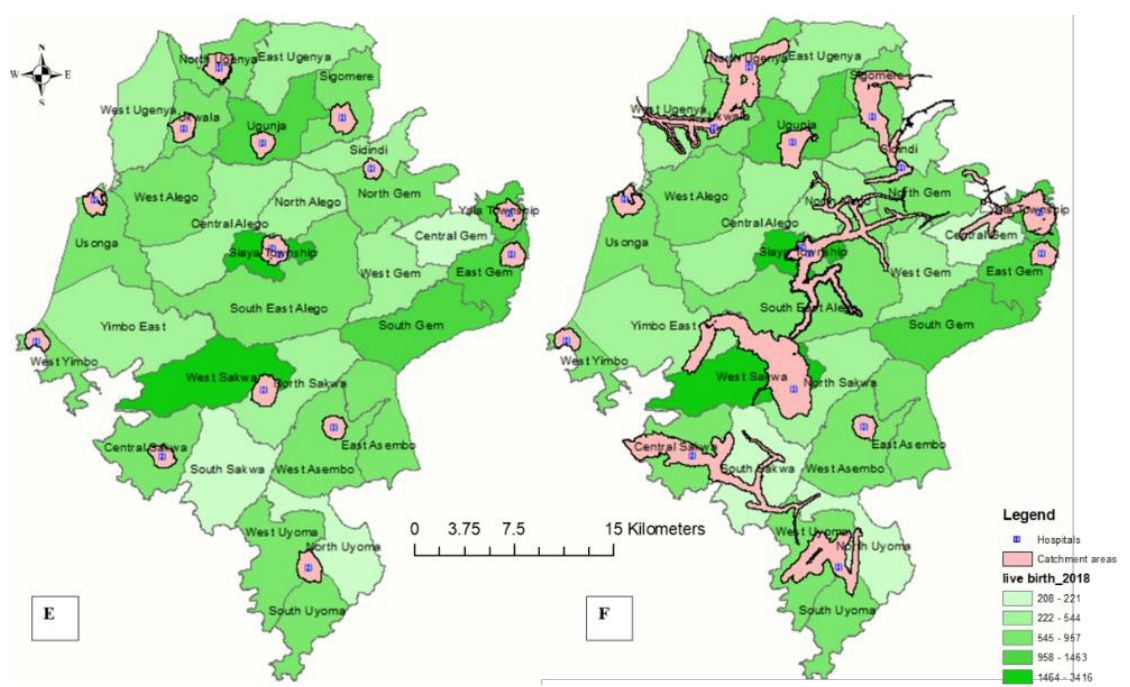

Figure 7. The distribution of hospital level geographic coverage across all wards in Siaya County $(\mathrm{E}=$ walking scenario; $\mathrm{F}=$ motorized scenario $)$.

\section{Likelihood of Delivering in a Particular Health Facility Type}

Zero-inflated Poisson model was used to model the statistical relationship between Geographic Coverage (independent variable) and Delivery by Skilled Birth Attendant Coverage (dependent variable) at the ward administrative level based on walking/motorbike traveling scenario, albeit disaggregated by facility type (dispensary, Health Centers or Hospital). Nevertheless, for both walking and/or motorbike traveling scenarios, a computed p-value $<0.05$ represented a statistically significant relationship between skilled birth attendance and geographic coverage of pregnant women across all facility types in Siaya County (see Tables 5-7 below). For instance, for every unit increase in the proportion of pregnant women who could reach a preferred dispensary with enough capacity to cover the demand within an hour of walking, the probability of skilled delivery coverage increased by $9.7 \%$. Yet this likelihood also increased slightly, albeit by $7 \%$ if the traveling scenario involved motorcycle transport.

Table 5. Showing the estimates of the slope parameter and corresponding levels of significance for dispensary facility type

\begin{tabular}{l|lllll} 
& Estimate & $\mathbf{2 . 5} \%$ & $\mathbf{9 7 . 5 \%}$ & $\operatorname{Pr}(>|\mathbf{z}|)$ \\
\hline count_disp_geog_walk & 1.0973035 & 1.0941537 & 1.1004624 & $<2 \mathrm{e}-16^{* * * *}$ \\
count_disp_geog_bike & 1.070097 & 1.067837 & 1.0723617 & $<2 \mathrm{e}-16^{* * * *}$
\end{tabular}

Moreover, for health centers, a unit increase in the proportion of pregnant women who could walk to such facilities with enough capacity to cover demand within an hour would result in a slightly higher increase in the probability of skilled delivery coverage by $16 \%$. Although such a likelihood would also increase by a smaller margin of $9 \%$ if the same proportion of 
pregnant women opted for motorcycle transport to reach the nearest health center with enough capacity to cover the demand as shown (in Table 6) below.

Table 6. The estimates of the slope parameter and corresponding levels of significance for health center facility type

\begin{tabular}{l|llll} 
& Estimate & $\mathbf{2 . 5} \%$ & $\mathbf{9 7 . 5} \%$ & $\operatorname{Pr}(>|\mathrm{z}|)$ \\
\hline count_health_centre_geog_walk & 1.1646251 & 1.1622717 & 1.166983 & $<2 \mathrm{e}-16$ *** \\
count_health_centre_geog_bike & 1.0903818 & 1.0891489 & 1.091616 & $<2 \mathrm{e}-16 * * *$
\end{tabular}

Hospitals, on the other hand, would experience the highest increase in the odds of skilled delivery coverage of about $34 \%$ for every unit increase in the proportion of pregnant women with access to such facility types with enough capacity to cover demand via walking scenario. This would be the case even if increasing the same proportion of geographic coverage based on the motorcycle scenario would only increase skilled delivery coverage by $13 \%$ as shown (in Table 7) below.

Table 7. Showing the estimates of the slope parameter and corresponding levels of significance for hospital facility type

\begin{tabular}{l|llll} 
& Estimate & $\mathbf{2 . 5} \%$ & $\mathbf{9 7 . 5 \%}$ & $\operatorname{Pr}(>|\mathbf{z}|)$ \\
\hline count_hosp_geogwalk & 1.3433355 & 1.3395178 & 1.3471640 & $<2 \mathrm{e}-16 * * *$ \\
count_hosp_geogbike & 1.1252405 & 1.1238829 & 1.126600 & $<2 \mathrm{e}-16 * * *$
\end{tabular}

\section{Discussion}

The current study demonstrates the feasibility of combining routine health facility data on total catchment area population with a least-cost algorithm based on Tanahashi's framework (1978) to evaluate health service (geographic) coverage at the ward administrative level, at least in Siaya County. More importantly is that the current summary statistics from the web based DHIS2 platform highlight the fact that almost half of all the wards in Siaya County were using less than $50 \%$ of their estimated skilled deliveries capacity. It is nevertheless important to understand that the estimated skilled delivery capacity of each ward was simply calculated by adding the total catchment area population of individual health facilities offering free maternity services without considering their physical accessibility. Yet a recent WHO sanctioned study in Malawi concluded that benchmarks that only consider density of emergency obstetric care (EmOC) facilities at the national or sub national level without taking accessibility and geographic coverage into account may not be appropriate (WHO, 2016b).

Moreover, the current findings also revealed that the Ministry of Health facility types were the most available (89\%). In particular, the estimated skilled delivery capacity at the dispensary facility types (41\%) was almost more than twice that of the hospital facility types (22\%) across all wards in Siaya County. Despite this, deliveries in hospitals facility types which open 24 hours a day, seven days a week, were over four times that of 
localized dispensary facility types which are usually closed by 5 p.m. and do not work during the weekends and on public holidays. Nevertheless, a most recent study on the barriers to formal health care seeking during pregnancy, childbirth, and postnatal period in Siaya County explained that facility closures during the night and weekends considerably increased the distance that had to be travelled beyond what had been planned, and it also restricted the transportation modes available (Ochieng \& Odhiambo, 2019). The same study thus recommended for the re-consideration of opening times of the health facilities in Siaya County, despite the unpredictability of labor emerging as the biggest deterrent to facility delivery, since most labor was reported to begin at night, at a time when there would be no means of transport to a better or farther health facility, therefore "forcing" the women to deliver at home (Ochieng \& Odhiambo, 2019).

In terms of geographic coverage, the current findings suggest that even if all pregnant women in Siaya County had access to motorcycle transportation, the geographic extent or catchment area of existing facility types offering free maternity health services would only increase by a modest $8-10 \%$ before reaching either their maximum allowable travel time $(60 \mathrm{~min})$ or population coverage capacity. This is despite the fact that 56 dispensaries, 21 health centers and 8 hospital facility types in Siaya County were estimated to be working above their capacity, and should thus be prioritized for upgrading. For instance, a recent study examining the implementation of the Free Maternity Services Policy in Kenya recommended that the Ministry of Health and County governments should conduct an assessment of the supply side capacity gaps of healthcare providers and make investments to build such capacity/ This should be done so as to match the increased demand under the free maternity services policy, which has since compromised the quality of maternity care (Tama et al., 2018). Similarly, a previous study on infrastructural challenges to better health in maternity facilities in rural Kenya explained that for communities where maternal and newborn deaths remain unacceptably high, the lack of obstetric capacity is not the only challenge to the low-tier health centers providing such services. Prerequisites such as reliable electricity, quality water and road networks, appropriate transportation facilities, and adequate qualified health personnel are equally essential for providing basic routine services which these facilities are designated to provide. Thus, improving infrastructure in predominantly rural settings, like Siaya County, also has the potential to reduce out-of-pocket costs for seeking skilled maternal and newborn care (Essendi et al., 2015).

More importantly, the present study also predicted the maximum likelihood of a skilled delivery based on the estimated geographic catchment area of different health facility types across Siaya County. The findings however suggest a very strong relationship between geographic coverage and 
the probability of a skilled delivery across all levels of care (hospitals, health centers or dispensaries), regardless of a walking or motorized traveling scenario. Nevertheless, the current findings in Siaya County also revealed a higher likelihood of a skilled delivery for every unit increase in the proportion of pregnant women within the spatial extent of a hospital or health center by walking, as compared to being within the geographic catchment area of a dispensary, even if they used motorcycle transport. The present findings were however synonymous with a fairly recent study on the Devolution of Public Health Care Services in Kenya, which found out that majority of existing facilities in the country have very old and dilapidated infrastructure constructed in the first quarter of 20th Century. Moreover, the situation was made worse with the non-targeted upgrading of lower tier facilities to higher levels, which do not necessarily conform to the required norms and standards with respect to human resources, infrastructure, and equipment (Okech, 2017). Moreover, a more recent GIS based study in Siaya County reaffirmed that increasing availability of low quality and non-comprehensive health facilities is not cost effective. This is because they are most likely to be bypassed and rendered redundant or grossly underutilized. Thus, county governments ought to prioritize improving quality and expanding services within existing health facilities (Nyangueso et al., 2018).

Nevertheless, a major caution for the current geographic coverage analysis, as well as for other studies using least cost surface models, is the neglect of financial accessibility and acceptability, the two other dimensions of accessibility to quality care, which have not been considered explicitly in the predominantly spatial framework of the current study. This limitation may hinder the complete assessment of accessibility to maternity services in Siaya County. However, the current study only focused on facilities offering free maternity services, despite the assumption of horizontal equity after disaggregation of maternity services by health facility type. Moreover, there was also the assumption of a closed system- where women do not seek services outside the study area. Yet in actual sense, pregnant women living near borders were likely to travel beyond the current administrative boundaries of Siaya County to seek maternity services. There were also minor limitations in terms of time discrepancies and different resolutions between the publicly available geospatial datasets, despite omission of the few privately-owned facilities that were not offering free maternity health services. Moreover, boat and travel by car were not analyzed, as the study area was restricted to the larger mainland Siaya County. This is also because rural women in such a low resource setting with poor road networks were less likely to rely on car travel. 


\section{Conclusion and Recommendations}

In conclusion, the current geographic coverage analysis has confirmed the robustness of using local routine health facility data to model the extent of catchment areas across health facility types, and to also evaluate its impact on maternity health services utilization. Moreover, it should be understood that the capacity and, by extension, quality of existing facilities offering free maternity health services positively affects a pregnant woman's likelihood of utilizing skilled delivery, regardless of proximity. The immediate implication is that policy makers need to leverage locally generated data on total facility catchment populations, in combination with existing spatial tools and GIS platforms, in order to selectively target underperforming health facilities, while precisely estimating the number and location of underserved pregnant women not covered by adequate maternity health services in Siaya County and beyond. Future research should also consider looking at the cost-implications of scaling up existing maternity health services, albeit based on local routine health facility data.

\section{Acknowledgments}

I am grateful to the Siaya County Health Department, Division of Reproductive Health and the entire County Health Management Team for their support.

\section{References:}

1. Banke-Thomas, A., Wright, K., \& Collins, L. (2019). Assessing Geographical Distribution And Accessibility Of Emergency Obstetric Care In Sub-Saharan Africa: A Systematic Review. Journal Of Global Health, 9(1).

2. Ebener, S., \& Stenberg, K. (2016). Investing The Marginal Dollar For Maternal And Newborn Health: Geographic Accessibility Analysis For Emergency Obstetric Care Services In Cambodia.

3. Essendi, H., Johnson, F. A., Madise, N., Matthews, Z., Falkingham, J., Bahaj, A. S., . . . Blunden, L. (2015). Infrastructural Challenges To Better Health In Maternity Facilities In Rural Kenya: Community And Healthworker Perceptions. Reproductive Health, 12(1), 103.

4. Frenz, P., \& Vega, J. (2010). Universal Health Coverage With Equity: What We Know, Don't Know And Need To Know. Paper Presented At The Background Paper For The Global Symposium On Health Systems Research.

5. Gok (2014). Kenya Service Availability And Readiness Assessment Mapping (SARAM) Report. In: Ministry Of Health Nairobi.

6. KDHS (2014). Kenya Demographic And Health Survey, 2014. 
7. Kieny, M. P., Bekedam, H., Dovlo, D., Fitzgerald, J., Habicht, J., Harrison, G., . . Mirza, Z. (2017). Strengthening Health Systems For Universal Health Coverage And Sustainable Development. Bulletin Of The World Health Organization, 95(7), 537.

8. Moh (2016). Kenya Reproductive, Maternal, Newborn, Child And Adolescent Health (RMNCAH) Investment Framework. In: Ministry Of Health Nairobi, Kenya.

9. Molla, Y. B., Rawlins, B., Makanga, P. T., Cunningham, M., Ávila, J. E. H., Ruktanonchai, C. W., . . . Dwivedi, V. (2017). Geographic Information System For Improving Maternal And Newborn Health: Recommendations For Policy And Programs. BMC Pregnancy

10. Childbirth, 17(1), 26.

11. Munoz, U. H., \& Källestål, C. (2012). Geographical Accessibility And Spatial Coverage Modeling Of The Primary Health Care Network In The Western Province Of Rwanda. International Journal Of Health Geographics, 11(1), 40.

12. Nyangueso, S. O., Hayombe, P. O., \& Owino, F. O. (2018). Spatial Equity In Devolved Healthcare: Is It Quality Or Quantity Causing Spatial Clustering In Maternal Health Utilization When Affordability Has Been Addressed? American Journal Of Geographic Information System, 7(3), 88-98.

13. O'Connell, T., \& Sharkey, A. (2013). Reaching Universal Health Coverage Through District Health System Strengthening: Using A Modified Tanahashi Model Sub-Nationally To Attain Equitable And Effective Coverage. UNICEF.

14. Ochieng, C. A., \& Odhiambo, A. S. (2019). Barriers To Formal Health Care Seeking During Pregnancy, Childbirth And Postnatal Period: A Qualitative Study In Siaya County In Rural Kenya. BMC Pregnancy

15. Childbirth, 19(1), 339.

16. Okech, T. C. (2017). Devolution Of Public Health Care Services In Kenya And Its Implication On Universal Health Coverage.

17. Ray, N., \& Ebener, S. (2008). Accessmod 3.0: Computing Geographic Coverage And Accessibility To Health Care Services Using Anisotropic Movement Of Patients. 7(1), 63.

18. Schmitz, M. M., Serbanescu, F., Kamara, V., Kraft, J. M., Cunningham, M., Opio, G., . . . Goodwin, M. M. (2019). Did Saving Mothers, Giving Life Expand Timely Access To Lifesaving Care In Uganda? A Spatial District-Level Analysis Of Travel Time To Emergency Obstetric And Newborn Care. 7(Supplement 1), S151S167. Doi:10.9745/GHSP-D-18-00366 \%J Global Health: Science And Practice 
19. Siaya County (2018). County Integrated Development Plan (CIDP), Siaya County 2018-2022.

20. Tama, E., Molyneux, S., Waweru, E., Tsofa, B., Chuma, J., \& Barasa, E. (2018). Examining The Implementation Of The Free Maternity Services Policy In Kenya: A Mixed Methods Process Evaluation. International Journal Of Health Policy Management, 7(7), 603.

21. Tanahashi, T. (1978). Health Service Coverage And Its Evaluation. Bulletin Of The World Health Organization, 56(2), 295.

22. Tangcharoensathien, V., Mills, A., \& Palu, T. (2015). Accelerating Health Equity: The Key Role Of Universal Health Coverage In The Sustainable Development Goals. BMC Medicine, 13(1), 101.

23. UNFPA, K. (2014). Counties With The Highest Burden Of Maternal Mortality. In: Retrieved From UNFPA Kenya: Http://Kenya. Unfpa. Org/News/Counties-Highest ....

24. WHO (2016). Investing The Marginal Dollar For Maternal And Newborn Health: Geographic Accessibility Analysis For Emergency Obstetric Care Services In Malawi. Retrieved From

25. Worldpop (2019, 2019/04/10/). Worldpop Retrieved From Https://Www.Worldpop.Org/Doi/10.5258/SOTON/WP00645

26. You, D., Hug, L., Ejdemyr, S., Idele, P., Hogan, D., Mathers, C., . . . Alkema, L. (2015). Global, Regional, And National Levels And Trends In Under-5 Mortality Between 1990 And 2015, With ScenarioBased Projections To 2030: A Systematic Analysis By The UN InterAgency Group For Child Mortality Estimation. The Lancet, 386(10010), 2275-2286. 